\title{
Eliminating useless portfolios in constrained financial economies
}

Zaier Aouani, Bernard Cornet

Economics Department, Nazarbayev University Library

\begin{abstract}
When financial investors' portfolio holdings are unconstrained, financial economies are assumed, w.l.o.g., to have no redundant assets. Indeed, eliminating redundant assets allows to replace the initial financial structure by an equivalent one, i.e., one that has the same consumption equilibria. Moreover, at the end of the process, absence of redundant assets guarantees that the set of admissible portfolio allocations is bounded, a fundamental property for the existence of equilibria. In the presence of institutional (exogenous) portfolio constraints, eliminating redundant assets is not innocuous anymore since bounded arbitrage may persist at equilibrium, the law of one price does not hold, and some zero-income portfolios may not be free. The goal of the paper is to replace the elimination of redundant assets by the elimination of useless portfolios, a process that eliminates in particular Werner useless portfolios, but needs to go beyond to obtain the boundedness of the set of admissible portfolio allocations at the end of the purification process. Moreover, the elimination process is carried out without affecting the set of consumption equilibria, hence replacing at each step the financial structure by an equivalent one.
\end{abstract}

Original language English

Pages (from-to) $\quad 1-32$

Number of pages 32

Journal Economic Theory

State Accepted/In press - Oct 212016

Aouani, Z., \& Cornet, B. (2016). Eliminating useless portfolios in constrained financial economies. Economic Theory, 1-32. DOI: 10.1007/s00199-016-1000-5 